\title{
Electronic states in valence and conduction bands of group-III nitrides: Experiment and theory
}

\author{
K. Lawniczak-Jablonska* \\ Institute of Physics, Polish Academy of Sciences, Al. Lotnikow 32/46, 02668 Warsaw, Poland \\ T. Suski and I. Gorczyca \\ High Pressure Research Center, Polish Academy of Sciences, ulica Sokolowska 29/37, 01142 Warsaw, Poland \\ N. E. Christensen \\ Institute of Physics and Astronomy, Aarhus University, C8000 Aarhus, Denmark \\ K. E. Attenkofer \\ HASYLAB, DESY, Notkestrasse 85, 22603 Hamburg, Germany \\ R. C. C. Perera, E. M. Gullikson and J. H. Underwood \\ Center for X-ray Optics, Lawrence Berkeley National Laboratory, Berkeley, California 94720 \\ D. L. Ederer \\ Tulane University, New Orleans, Louisiana 70118 \\ Z. Liliental Weber \\ Lawrence Berkeley National Laboratory, MIS 6212031 Berkeley, California 94720 \\ (Received 21 July 1999; revised manuscript received 29 November 1999)
}

\begin{abstract}
A comprehensive study of the electronic structure of group-III nitrides (AlN, GaN, InN, and BN) crystallizing in the wurtzite, zinc-blende, and graphitelike hexagonal (BN) structures is presented. A large set of the $\mathrm{x}$-ray emission and absorption spectra was collected at the several synchrotron radiation facilities at installations offering the highest possible energy resolution. By taking advantage of the linear polarization of the synchrotron radiation and making careful crystallographic orientation of the samples, the bonds along $c$ axis $(\pi)$ and "in plane", $(\sigma)$ in the wurtzite structure could be separately examined. Particularly for AlN we found pronounced anisotropy of the studied bonds. The experimental spectra are compared directly with ab initio calculations of the partial density of states projected on the cation and anion atomic sites. For the GaN, AlN, and $\mathrm{InN}$ the agreement between structures observed in the calculated density of states (DOS) and structures observed in the experimental spectra is very good. In the case of hexagonal BN we have found an important influence of insufficient core screening in the X-ray spectra that influences the DOS distribution. The ionicity of the considered nitrides is also discussed.
\end{abstract}

\section{INTRODUCTION}

The group-III nitrides ( $\mathrm{GaN}$, AlN, InN, and $\mathrm{BN}$ ) are widegap refractory semiconductors with applications as basic materials in optoelectronic devices operating in the visible/ ultraviolet spectral range as well as in high-temperature and high-power microelectronic devices. ${ }^{1,2}$ However, the understanding of the basic physical properties leading to applications is still not satisfactory. One of the reasons consists of insufficient knowledge of the electronic band structure of the considered semiconductors being somewhat "untypical" comparing to the "classic" III-V materials. Apart from BN the nitrides crystallize in the thermodynamically stable wurtzite structure and in the metastable sphalerite (zincblende) structure (cubic). BN has hexagonal and cubic phases similar to the graphite and diamond phases of carbon. Recently, several other metastable structures have been identified (rhombohedral, wurtzite, simple cubic, and turbostratic), ${ }^{3}$ and also a fullerenelike structure has been synthesized and characterized. ${ }^{4}$ The coordination number is the same, 4 , in all structures examined here. There are, nevertheless, some differences in physical properties of these nitride phases as is shown in Table I, where the most important physical parameters of nitrides are summarized. In all materials and structures nitrogen as an anion leads to the formation of very short, strong bonds (see Table I). Comparing to other III-V semiconductors, we find the bond lengths in nitrides approximately $20 \%$ shorter (e.g., $1.95 \AA$ for wurtzite $\mathrm{GaN}$ in comparison with $2.7 \AA$ in GaAs) and the ionicity roughly two times higher.

Several theoretical studies of III-V nitrides have been published $^{8-11}$ but surprisingly, relatively few experimental studies of the band structure have been carried out ${ }^{8,10,12}$ except for BN. Only recently some X-ray studies concerning mainly GaN have been published. ${ }^{13-16}$ The shape of the $\mathrm{x}$-ray spectra is a fingerprint of the particular chemical bond and makes it possible to identify the chemical nature of the bond formed by selected kind of atoms in different compounds. Photon absorption and emission involves an optical transition between electronic states of the atom in the 
TABLE I. Physical properties of nitrides. Unless otherwise indicated data for nitrides are from Ref. 17.

\begin{tabular}{|c|c|c|c|c|c|}
\hline \multirow[t]{2}{*}{ Material } & \multirow{2}{*}{$\begin{array}{c}\text { Band gap } \\
(\mathrm{eV})\end{array}$} & \multirow{2}{*}{$\begin{array}{l}\text { Lattice constants }(a, c) \\
\text { and bond lenghts }(b, d) \\
(\AA)\end{array}$} & \multirow{2}{*}{$\begin{array}{l}\text { Ionicity } \\
\text { (Phillips) }\end{array}$} & \multicolumn{2}{|c|}{ Ratio of states nos. } \\
\hline & & & & Valence & Conduc. \\
\hline \multirow[t]{5}{*}{ GaN wurtzite } & 3.5 & $a=3.1892$ & 0.43 & $\mathrm{~N} / \mathrm{Ga}=3.5$ & 0.67 \\
\hline & & $c=5.1850^{\mathrm{a}}$ & & $\mathrm{Ga} / \mathrm{N}=0.28$ & 1.49 \\
\hline & & $c / a=1.626$ & & & \\
\hline & & $b / c=0.377$ & & & \\
\hline & & $b=1.955 ; d=1.9486$ & & & \\
\hline \multirow[t]{2}{*}{ GaN cubic } & 3.3 & $a=4.511^{\mathrm{b}}$ & & $\mathrm{N} / \mathrm{Ga}=3.4$ & 0.98 \\
\hline & & $b=2.1923$ & & $\mathrm{Ga} / \mathrm{N}=0.29$ & 1.02 \\
\hline \multirow[t]{5}{*}{ AlN wurtzite } & 6.1 & $a=3.1106$ & 0.44 & $\mathrm{~N} / \mathrm{Al}=4.4$ & 0.64 \\
\hline & & $c=4.9795^{\mathrm{c}}$ & & $\mathrm{Al} / \mathrm{N}=0.23$ & 1.56 \\
\hline & & $c / a=1.601$ & & & \\
\hline & & $b / c=0.3821$ & & & \\
\hline & & $b=1.90 ; d=1.807$ & & & \\
\hline \multirow[t]{2}{*}{ AlN cubic } & $4.5-6.2$ & $a=4.38$ & & $\mathrm{~N} / \mathrm{Al}=4.6$ & 0.80 \\
\hline & & $b=2.1287$ & & $\mathrm{Al} / \mathrm{N}=0.22$ & 1.25 \\
\hline \multirow[t]{3}{*}{ InN wurtzite } & 1.9 & $a=3.54$ & 0.57 & $\mathrm{~N} / \mathrm{In}=4.4$ & 0.96 \\
\hline & & $c=5.70$ & & $\mathrm{In} / \mathrm{N}=0.23$ & 1.04 \\
\hline & & $c / a=1.610$ & & & \\
\hline \multirow[t]{2}{*}{ InN cubic } & $1.9-2.0$ & $a=4.98$ & & $\mathrm{~N} / \mathrm{In}=4.0$ & 0.97 \\
\hline & & $b=2.4203$ & & $\mathrm{In} / \mathrm{N}=0.25$ & 1.03 \\
\hline \multirow[t]{5}{*}{ BN hex. } & $5.4-5.8$ & $a=2.54$ & 0.36 & $\mathrm{~N} / \mathrm{B}=2.2$ & 0.85 \\
\hline & & $c=4.17$ & & $\mathrm{~B} / \mathrm{N}=0.45$ & 1.18 \\
\hline & & $c / a=1.64$ & & & \\
\hline & & $b / c=0.375$ & & & \\
\hline & & $b=1.156 ; \mathrm{d}=1.556$ & & & \\
\hline \multirow[t]{2}{*}{ BN cubic } & $6.1-6.4$ & $a=3.6160(3)$ & & $\mathrm{N} / \mathrm{B}=1.96$ & 0.80 \\
\hline & & $b=1.7573$ & & $\mathrm{~B} / \mathrm{N}=0.51$ & 1.25 \\
\hline
\end{tabular}

${ }^{\mathrm{a}}$ Reference 6 .

${ }^{\mathrm{b}}$ Reference 7 .

${ }^{\mathrm{c}}$ Reference 5.

sample. This allows the interpretation of the experimental spectra in terms of the density of the occupied states (valence band) for emission and density of unoccupied states (conduction band) for absorption spectra. The electron transition is governed by dipole selection rule and hence the investigated electronic states are also "orbital resolved." The linear polarization of the synchrotron radiation allows a separation of crystallographic-direction-dependent contributions from various constituents to the band states and thus a description of the anisotropy of chemical bonding. ${ }^{15}$

In the present paper a systematic experimental and theoretical study of atom- and orbital-projected partial density of states (DOS) for the group-III nitrides is reported. We compare the energy distribution of electronic states in valence and conduction bands as calculated by means of the linear muffin-tin orbital (LMTO) method with x-ray emission and absorption spectra. We do not attempt to calculate the DOS with the broadening functions to account for the lifetime of the involved core levels and spectrometer resolving functions to mimic exactly the relative intensity between peaks observed in spectra. Instead, the direct comparison with the appropriate "raw" DOS is presented to indicate the extend to which we can get direct information about allowed electron states by consideration the positions of the maxima and minima of the intensity in spectra. The influence of the core level width and spectrometer broadening function on the spectral structures is also discussed.

The good agreement between structures observed in spectra and structures in the calculated DOS allows for consistent analysis of the results. We compare the amounts of bonding and antibonding states near the band edges for different choices of cations and crystal structures. Since the device applications are based mainly on wurtzite-type nitrides, particular attention is paid to this phase. In particular, we examine for wurtzite structure the level of anisotropy in the formed chemical bonds along the $c$ axis ( $\pi$ bond) and "in plane,' i.e., slightly inclined with respect to the $c$ plane ( $\sigma$ bonds). These two kinds of bonds, $\pi$ and $\sigma$, can be connected directly with $b$ and $d$ bond lengths, respectively. Their values as obtained by x-ray diffraction measurements ${ }^{17}$ are given in Table I.

Finally, we examine the amount of electronic states available for optical transition as a function of crystal structure, crystallographic direction, and the presence of $d$ cation semi- 
core states. The role of the cation semicore $d$ states in the determination of different properties of group-III nitrides has been discussed by several authors. ${ }^{8}$ In this context the hybridization between $d$ and $p$ states for different cations and nitrogen is investigated. The $d-p$ interaction can affect the valence-band edge and may influence magnitude of the fundamental gap. It has been suggested that the cation$4 d$-anion- $p$ hybridization is stronger in AlN than in GaN.

\section{EXPERIMENT}

The $\mathrm{x}$-ray absorption and emission measurements for lines with energy up to $600 \mathrm{eV}$ were performed at the Lawrence Berkeley National Laboratory (LBNL) Advanced Light Source (ALS) at the beam lines 6.3.2 and 8.0, respectively. ${ }^{18}$ In the case of $\mathrm{x}$-ray absorption the total photocurrent measurement technique was applied for recording of the spectra. The energy resolution $\Delta E$ for the 1200 line/mm grating employed for nitrogen $K$ edge (with a 50 $\mu \mathrm{m}$ exit slit) was close to $0.16 \mathrm{eV}$. The $M_{2,3}$ edge of $\mathrm{Ga}, L_{2,3}$ edge of $\mathrm{Al}$, and $K$ edges of $B$, were measured using a 300 line/mm grating with a $50 \mu \mathrm{m}$ exit slit, which results in resolution $0.2 \mathrm{eV}, 0.04 \mathrm{eV}$, and $0.03 \mathrm{eV}$, respectively. Measurements of $L_{2,3}$ edges of $\mathrm{Ga}$ and $K$ edges of $\mathrm{Al}$ were carried out at the SA32 station of the SuperAco ring, LURE. The $L_{2,3}$ edges of $\mathrm{Ga}$ were measured using a $\mathrm{Be}(1010)$ monochromator, which provides an energy resolution of $\Delta E \sim 0.6 \mathrm{eV}$, and the $K$ edges of $\mathrm{Al}$ were measured with a quartz (1010) monochromator with a resolution of $\Delta E \sim 0.6$ $\mathrm{eV}$. The $\mathrm{Ga} K$-edge data were recorded using a $\mathrm{Si}$ (111) monochromator at the D-21 station, DCI ring, LURE and with higher resolution using the four crystalline Si monochromators in HASYLAB, station A1. At this station also the In $L$-edge spectra were measured.

The spectra of wurtzite structure samples were recorded at different angles between the single crystalline sample surface (its $c$ plane) and the polarization vector of synchrotron radiation. At normal incidence the polarization vector e was parallel to the sample surface, and electrons were excited in the direction of the $c$ plane probing three $\sigma$ bonds out of the four forming a tetrahedral coordination. At grazing incidence the polarization vector e formed a small angle with the $c$ axis (which is normal to the sample surface). Therefore the states localized along the $c$ axis (single $\pi$ bond) should predominate the spectra. In the case of large anisotropy (AIN) the spectra were also measured at an angle $46^{\circ}$ to the surface where both bonds should be probed. For epitaxial layers the photocurrent was measured from an isolated sample. In the hard x-ray region the intensity at $I_{0}$ was monitored by an ionization chamber with proper pressure and mixture of gases in the soft energy region by the photocurrent generated at the focusing mirror. Additional transmission measurements were performed on $\mathrm{GaN}$ and $\mathrm{InN}$ powders to test the influence of surface oxides on the absorption. The spectra registered in transmission and at the angle of $46^{\circ}$ were identical. Therefore, the influence of the surface contamination on the spectra from layers was negligible.

High-resolution x-ray emission spectra were recorded at the ALS station 8.0 on the $5.0 \mathrm{~cm}$ period undulator beamline with a spherical grating monochromator operating between $70 \mathrm{eV}$ and $1200 \mathrm{eV} .{ }^{19}$ The $K$-emission spectra of $\mathrm{N}$ were measured using the 1000 line/mm, $10 \mathrm{~m}$ radius grating. At the $400 \mathrm{eV}$ energy the resolution of the spectrometer was approximately $0.8 \mathrm{eV}$ and at the $200 \mathrm{eV}$ about $0.5 \mathrm{eV}$. The energy of the excitation radiation was set high above resonant excitation.

The samples of polycrystalline, hexagonal, and cubic BN and wurtzite $\mathrm{GaN}$ and $\mathrm{InN}$ were commercially available powders (with particles size smaller than $20 \mu \mathrm{m}$ ). For these samples we were not able to measure polarization resolved spectra. The wurtzite $\mathrm{GaN}$ and $\mathrm{InN}$ films were grown by molecular beam epitaxy (MBE) on sapphire substrate. The nucleation and growth of the wurtzite AIN and cubic GaN and InN films were performed in the MBE system on singular (001) $n+$ Sn-doped GaAs substrates. The thicknesses of the epitaxial layers used in our measurements were between 0.5 and $1 \mu \mathrm{m}$. Transmission electron microscopy (TEM) cross-section and x-ray diffraction studies have clearly shown formation of single wurtzite or cubic phases.

\section{THEORY}

The band structure and DOS for the all group-III nitrides in wurtzite and zinc-blende structures are calculated by means of the LMTO method in its scalar relativistic form, ${ }^{20}$ in conjunction with the local density approximation (LDA) to the density functional theory. Here we apply the simplest version of the LMTO method, the atomic-sphere approximation (ASA) but with the "combined correction", terms ${ }^{20}$ incorporated. The ASA version applies spherically symmetrized charge distributions and potentials in atomic spheres, i.e., space filling (and thus slightly overlapping) spheres. Consequently, calculations for the semiconductors must include so-called "empty spheres" located in the interstitial positions, i.e., atomic spheres without "nuclear', charge. ${ }^{21}$ Each unit cell in the cubic (zinc-blende) structure contains two real atoms (cation and anion) and two empty spheres. In the wurtzite structure we have eight atoms in the unit cell (four "real"' and four "empty").

In the calculations presented here we have used the "standard" basis set, which includes partial waves of $s, p$, and $d$ character on each atomic and interstitial site to give a total of 36 LMTO orbitals per cubic unit cell. The "semicore" shallowed $d$ states in $\mathrm{GaN}$ and $\mathrm{InN}$ are treated as fully relaxed band states. This is especially important for $\mathrm{GaN}$, as it was already shown. ${ }^{8}$ Energy eigenvalues and wave functions were obtained at 95 (140) $\mathbf{k}$ points in the irreducible part of the Brillouin zone. The densities of states, normalized to the unit cell, were calculated by means of the tetrahedron technique. $^{22}$

The calculations for wurtzite structure were performed under the assumption that the crystal structure was "ideal," meaning that the $c / a$ ratio was taken as equal to 1.633 and the internal bond-length parameter $u$ was 3/8. Experimental $c / a$ values are 1.627, 1.600, and 1.612 for GaN, AlN and InN, respectively. ${ }^{23} \mathrm{X}$-ray diffraction measurements ${ }^{24}$ have shown that $u=0.377$ for GaN and 0.3821 for AlN (Table I). The calculations for hexagonal $\mathrm{BN}$ were performed by a slightly different method, the full-potential version ${ }^{25}$ of the LMTO scheme, where the full nonspherical shapes of potentials and charge distributions are taken into account. 

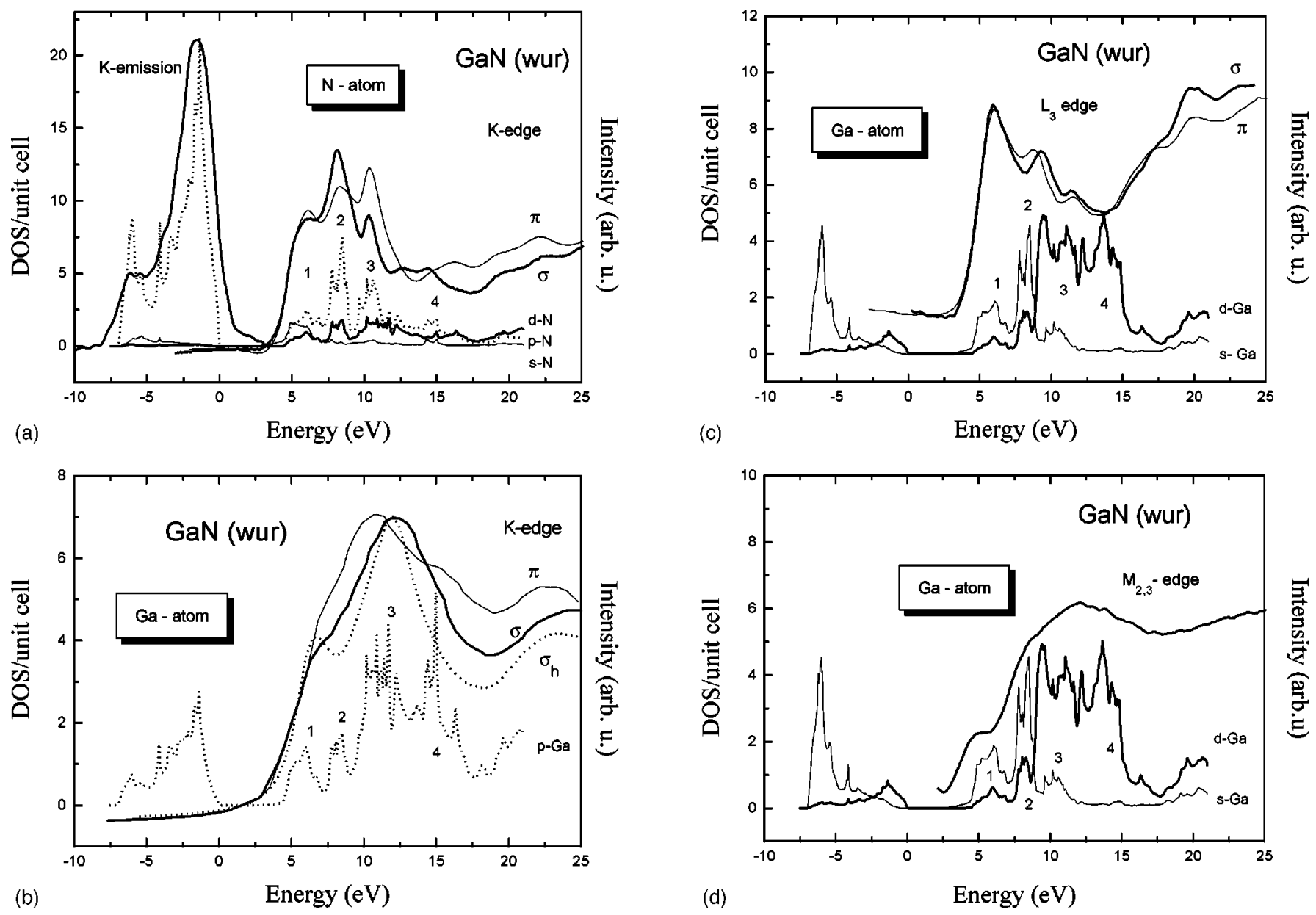

FIG. 1. The PDOS ( $s$ thin line; $p$, dotted line; $d$, thick line) as calculated for the wurtzite structure of GaN. (a) Projected on the N atom and compared with $\mathrm{N} K$ emission and absorption spectra for $\pi$ and $\sigma$ polarization geometry. (b) Projected on the Ga atom: $p$ PDOS compared with $\mathrm{Ga} K$ absorption spectra measured for $\pi$ and $\sigma$ polarization geometry. Additionally the high-resolution $\sigma$ spectrum is shown (dotted line). (c) Projected on the Ga atom: $s$ and $d$ PDOS compared with Ga $L_{3}$ absorption spectra measured for $\pi$ and $\sigma$ polarization geometry. (d) Projected on the Ga atom: $s$ and $d$ PDOS compared with the Ga $M_{2,3}$ absorption spectrum.

\section{RESULTS AND DISCUSSION}

Figures 1 through 8 show the partial DOS (PDOS), as obtained by the LMTO method and projected onto the atomic sites, together with the experimental $\mathrm{x}$-ray emission and absorption spectra, for wurtzite and cubic GaN, AlN, $\mathrm{InN}$, and for hexagonal and cubic BN. Linearly polarized synchrotron radiation irradiating well-oriented samples of wurtzite $\mathrm{GaN}$, AlN, and $\mathrm{InN}$ allowed us to precisely examine the anisotropy of the bonds formed in this structure. The reference energies of the experimental spectra were adjusted to give the best overall agreement of the characteristic spectral features of the theoretical DOS functions. The energy zero corresponds to the valence-band (VB) edge. On the left vertical axis the calculated total or partial $(s, p, d)$ DOS per unit cell at the chosen atom is indicated, which allows us to compare the absolute number of the available states. Note that the number of atoms in the wurtzite unit cell is two times larger. Therefore, for direct comparison the number of states in the cubic structure should be multiplied by a factor 2. On the right vertical axis the intensity of experimentally measured spectra in arbitrary units is shown. The spectra measured at different angles were normalized to the equal intensities before the onset of edge and far above the con- duction band (CB) edge where no polarization dependence is expected. In the last two columns of the Table I ratios of the number of states located at cation and anion sites for VB and $\mathrm{CB}$ as calculated from theoretical DOS are given.

\section{A. GaN}

The GaN absorption spectra have been already discussed, ${ }^{13-15}$ but importantly, with the spectra recorded for different polarization we were able differentiate contributions of $\sigma$ and $\pi$ bonds to the particular structures in the density of states. Figure 1(a) contains the experimental emission and absorption spectra at the $K$ edge of nitrogen, which correspond to contribution of $p$ states of $\mathrm{N}$ to the valence and conduction bands, respectively. Additionally, calculated PDOS representing contributions of $s, p$, and $d$ states of $\mathrm{N}$ are shown. The states are localized in easily seen subbands with energy positions that agree with the maxima in observed emission and absorption spectra. The crystal field splits the valence states into two subbands and the conduction states, in the considered energy range, into four separate subbands. As one can see the $p$ states dominate over all other states. Note, however, that the conduction-band edge has also a significant contribution of nitrogen $s$ states. A similar- 
ity of the measured spectrum with the calculated $p$ DOS supports the dominant character of $p$ states in the valence and conduction bands. Considering the anisotropy of $p$ states as seen by contributions from $\pi$ and $\sigma$ bond states (measured by different polarizations of synchrotron beem) some moderate differences in peak intensities and in position of peak number 4 can be noticed.

For the $\mathrm{Ga}$ atom the absorption spectra have been measured only [Figs. 1(b,c,d)]. In Fig. 1(b) $K$-edge absorption spectra $(\pi, \sigma$, and high-resolution $\sigma)$ in comparison with calculated $p$ PDOS are shown. As in the case of nitrogen [Fig. 1(a)], one can notice the existence of four subbands in the calculated PDOS corresponding to the main features of the measured absorption spectra. However, the anisotropy of states distribution is more pronounced in comparison with nitrogen $\pi$ bonds. The maximum of $\pi \mathrm{Ga}$ bond states is now $2 \mathrm{eV}$ closer to the $\mathrm{CB}$ edge than the maximum of $\sigma$ bond states. Also the second and fourth maxima consist of predominately $\pi$ bond states. Consequently, in the $c$ direction for the indicated energies there are much more available states than "in plane." The observed anisotropy can influence the optical and transport properties in plane and in the $c$ direction.

In Figs. 1(c) and 1(d) the $L_{3}$ and $M_{2,3}$ absorption spectra of Ga are shown together with the calculated PDOS of $s$ and $d$ symmetry. We observe in the case of the $L_{3}$ edge three well-resolved peaks that correspond preferentially to the three peaks in $s$ PDOS. The observed anisotropy of the $L_{3}$ edge is much smaller than that found at the $K$ edge, which confirms the $s$ character of projected states. However, the similarity of the CB states of $s$ and $d$ symmetry indicates strong hybridization of these states up to $12 \mathrm{eV}$. The $d$ PDOS obtained from the calculations should produce in the measured spectrum a broad maximum between 13 and $15 \mathrm{eV}$, but it is not observed, suggesting that the matrix element for $2 p$ $4 d$ transitions is small. Regarding the $M_{2,3}$ spectra [Fig. $1(\mathrm{~d})$ ] one can notice that though the spectral resolution is high $(0.04 \mathrm{eV})$ and the natural width of the $3 p$ levels is small, only the first minimum and another one around 17.5 $\mathrm{eV}$ are well resolved. This may be due to overlap of the spin-orbit-split bands; however, a close similarity of the measurements with the combined $s+d$ DOS suggests that the $3 p-4 d$ transition rate is comparable with the $3 p-4 s$ transition rate. Comparing Figs. 1(c) and 1(d) we can conclude that the shape of $L_{3}$ edge is dominated by $s$ conduction states, whereas the shape of $M_{2,3}$ edge by $s$ and $d$ states. The observed differences in the shape of $L_{3}$ and $M_{2,3}$ edges indicate the differences in the transition matrix for $2 p$ and $3 p$ core levels to $4 s$ and $4 d$ conduction states.

The results for $\mathrm{GaN}$ in the cubic structure are presented in Fig. 2. The general features of calculated PDOS and measured spectra, especially at the $\mathrm{N}$ site, are very similar in the cubic and wurtzite cases. Some differences are in the distribution of $s$ and $d$ states at Ga site-there are decidedly fewer states of Ga $d$ symmetry in the cubic structure. However, the general shape of the $\mathrm{Ga} L_{3}$ edge has the same character as in the wurtzite phase, which confirms that the transition matrix to $d$ states is small.

Summarizing, the main features of wurtzite and cubic GaN structures are (i) the dominant character of $p$ states, particularly at the $\mathrm{N}$ site, for both VB and $\mathrm{CB}$, (ii) the domi-
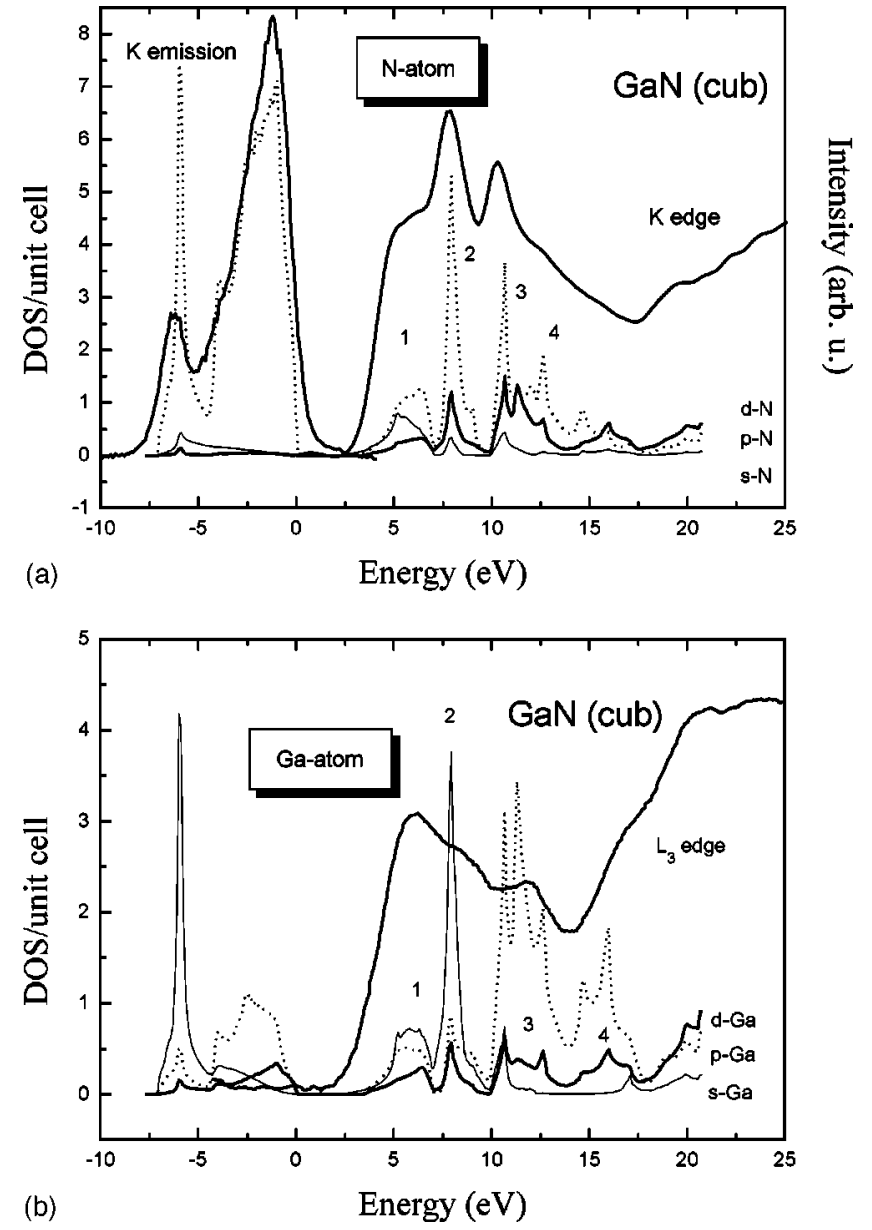

FIG. 2. The PDOS ( $s$ thin line; $p$, dotted line, $d$, thick line) as calculated for the cubic structure of GaN. (a) Projected on the $\mathrm{N}$ atom and compared with $\mathrm{N} K$ emission and absorption spectra. (b) Projected on the $\mathrm{Ga}$ atom and compared with the $\mathrm{Ga} L_{3}$ absorption spectrum.

nant contribution of $s$ states of Ga at the bottom of the VB for cubic structure and smaller contribution of these states for wurtzite structure, and (iii) significant hybridization $p$ $d(\mathrm{Ga})$ at valence- and conduction-band edges.

\section{B. AlN}

Although the AlN wurtzite PDOS and the measured x-ray spectra (presented in Fig. 3) are essentially similar to those for $\mathrm{GaN}$, some differences exist. One pronounced difference is that at the AlN CB edge the $d$ states of cation dominate over $s$ states, contrary to the GaN case. In VB and $\mathrm{CB}$ the $\mathrm{Al}$ states of $p$ as well as $d$ symmetry are located at the same energy as the density maximum of $\mathrm{N}$ states of $p$ symmetry, reflecting the strong hybridization of these states. As it was already mentioned in the Introduction, $p-d$ hybridization may be one of the reasons for the much larger energy gap of AlN in comparison with GaN. Considering the higher-energy part of $\mathrm{CB}$ (up to $13 \mathrm{eV}$ ) there is a smaller amount of $d$ states and bigger contribution of $s$ states of the cation [Fig. 3(c)], but, as in $\mathrm{GaN}$, the similarity of the states of $s$ and $d$ symmetry indicates strong hybridization of these states. The second, even more pronounced difference is connected with $\sigma$ - and $\pi$-bond distribution. In AlN the anisotropy of the $p$ conduc- 

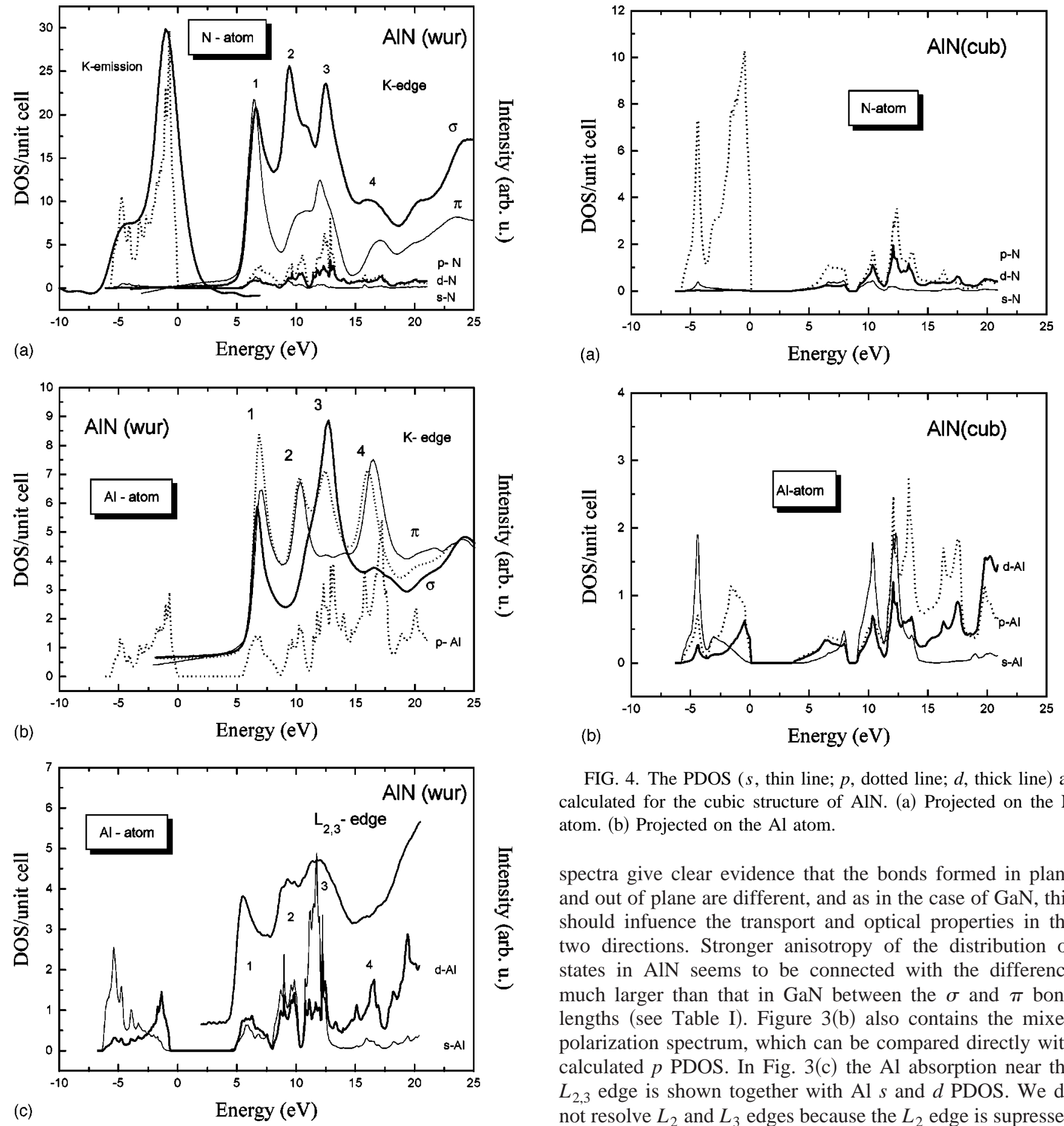

FIG. 3. The PDOS ( $s$ thin line; $p$, dotted line; $d$, thick line) as calculated for the wurtzite structure of AlN. (a) Projected on the $\mathrm{N}$ atom and compared with $\mathrm{N} K$ emission and absorption spectra measured for $\pi$ and $\sigma$ polarization geometry. (b) Projected on the Al atom: $p$ PDOS compared with Al $K$ absorption spectra measured for $\pi$ and $\sigma$ polarization geometry, additionally the mixed-bond spectrum is shown (dotted line). (c) projected on the Al atom: $s$ and $d$ PDOS compared with the $\mathrm{Al} L_{3}$ absorption spectrum.

tion states distribution is very pronounced at the anion as well as at the cation site [Figs. 3(a) and 3(b)]. The second peak in the CB localized at the $\mathrm{N}$ atom has a $\sigma$-bond origin, whereas only the states with $\pi$-bond character contribute to this peak at $\mathrm{Al}$ site. The reported polarization-dependent

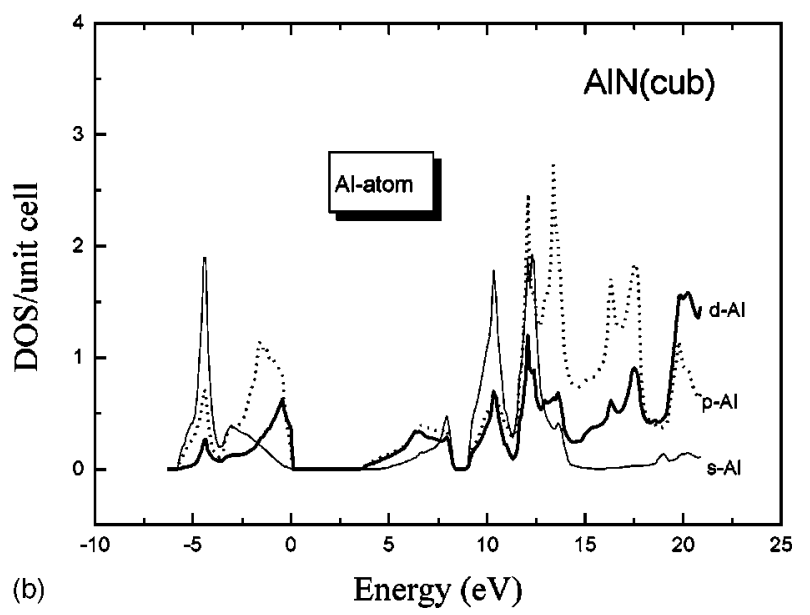

FIG. 4. The PDOS ( $s$, thin line; $p$, dotted line; $d$, thick line) as calculated for the cubic structure of AlN. (a) Projected on the N atom. (b) Projected on the Al atom.

spectra give clear evidence that the bonds formed in plane and out of plane are different, and as in the case of GaN, this should infuence the transport and optical properties in the two directions. Stronger anisotropy of the distribution of states in AlN seems to be connected with the difference much larger than that in $\mathrm{GaN}$ between the $\sigma$ and $\pi$ bond lengths (see Table I). Figure 3(b) also contains the mixed polarization spectrum, which can be compared directly with calculated $p$ PDOS. In Fig. 3(c) the Al absorption near the $L_{2,3}$ edge is shown together with $\mathrm{Al} s$ and $d$ PDOS. We do not resolve $L_{2}$ and $L_{3}$ edges because the $L_{2}$ edge is supressed by a Koster-Kronig Auger transition.

We did not have access to sufficient-quality samples of cubic AIN, and therefore only the calculated DOS are presented in Figs. 4(a) and 4(b). Generally, the PDOS is similar to the case of wurtzite AlN. The $p$ states dominate at the CB edge, as in wurtzite case, but now this edge is decidedly less sharp.

\section{C. $\mathbf{I n N}$}

As one can see from the Fig. 5 the calculated PDOS distribution and experimental spectra for $\mathrm{InN}$ resemble those for $\mathrm{GaN}$. In particular, the $p$-PDOS distribution and the general shape of the $K$-edge spectrum at $\mathrm{N}$ and at the cation site are very similar. Also the anisotropy of $\pi$ and $\sigma$ bonds is not so 

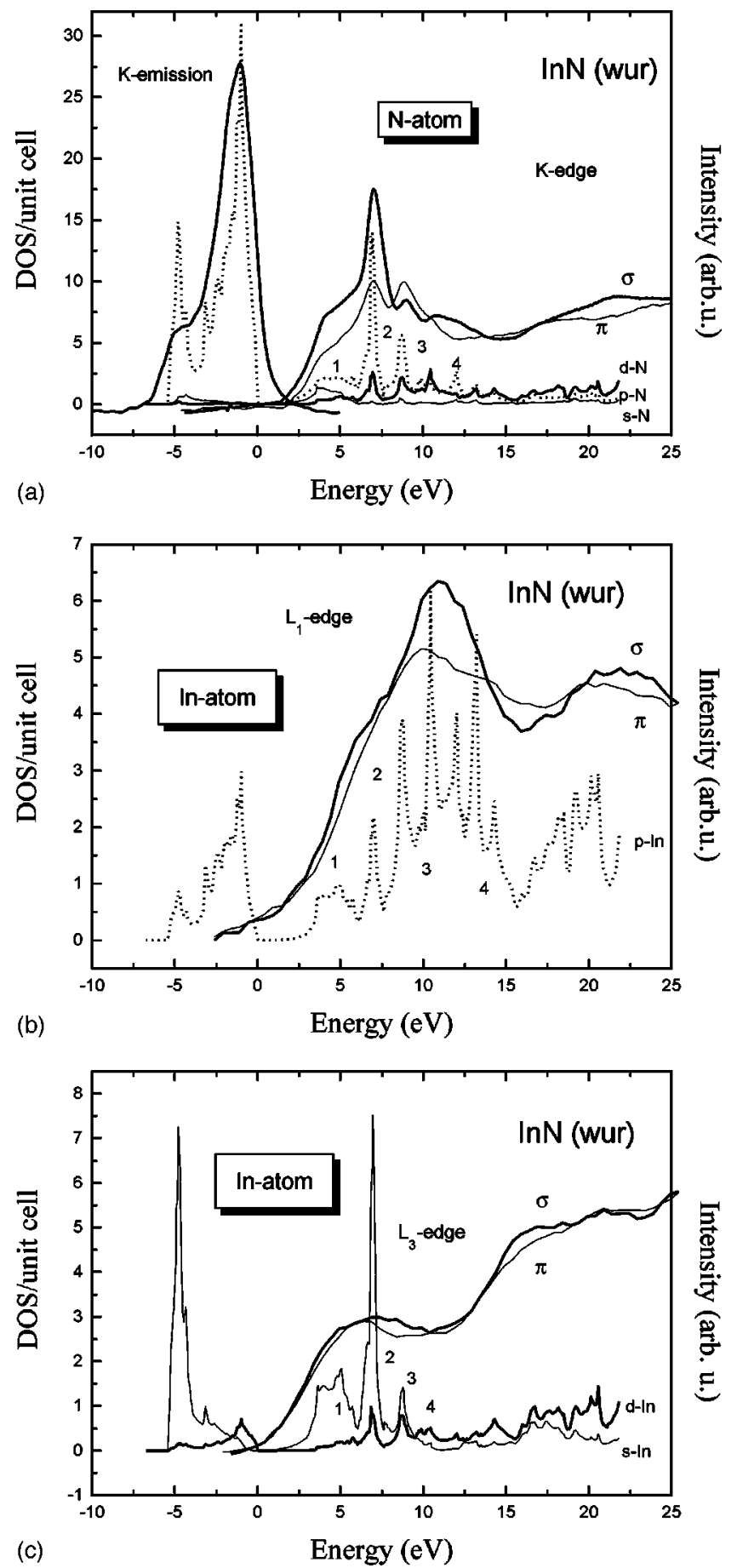

FIG. 5. The PDOS ( $s$, thin line; $p$, dotted line; $d$, thick line) as calculated for the wurtzite structure of InN. (a) Projected on the N atom and compared with $\mathrm{N} K$ emission and absorption spectra measured for $\pi$ and $\sigma$ polarization geometry. (b) projected on the In atom: $p$ PDOS and $L_{1}$ absorption spectra for $\pi$ and $\sigma$ polarization geometry. (c) Projected on the In atom: $s$ and $d$ PDOS and $L_{3}$ absorption spectra for $\pi$ and $\sigma$ polarization geometry.

strong as in AlN, but comparable with the GaN case. On the other hand, only in the case of InN do we observe at the $\mathrm{CB}$ edge a shift in the binding energy of the $\pi$ bond with respect to the $\sigma$ bond. At the $\mathrm{N}$ site this shift is about $0.8 \mathrm{eV}$ [Fig. 5(a)]. A similar effect, although less pronounced, is seen for the $L$ edge at In site [Figs. 5(b) and 5(c)]. It suggests an anisotropy of the InN band gap around the $\Gamma$ point. In con-
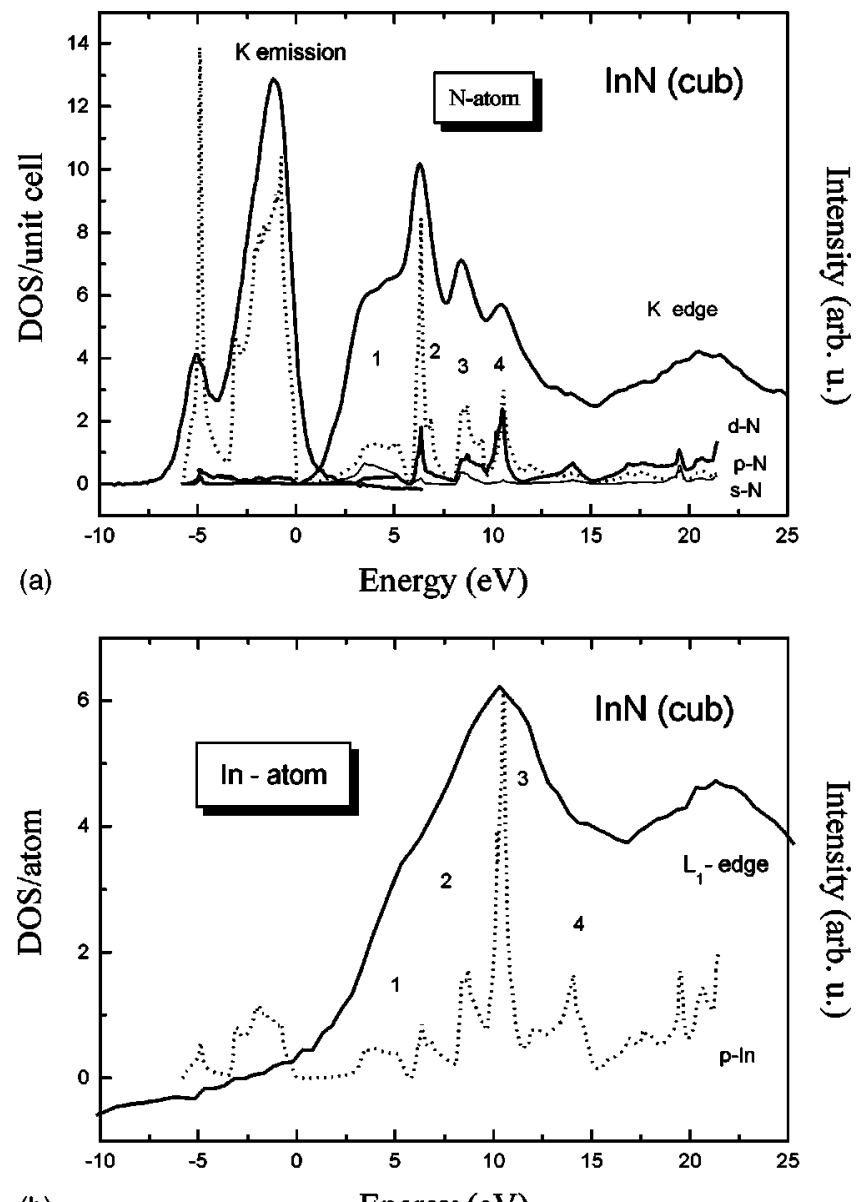

(b)

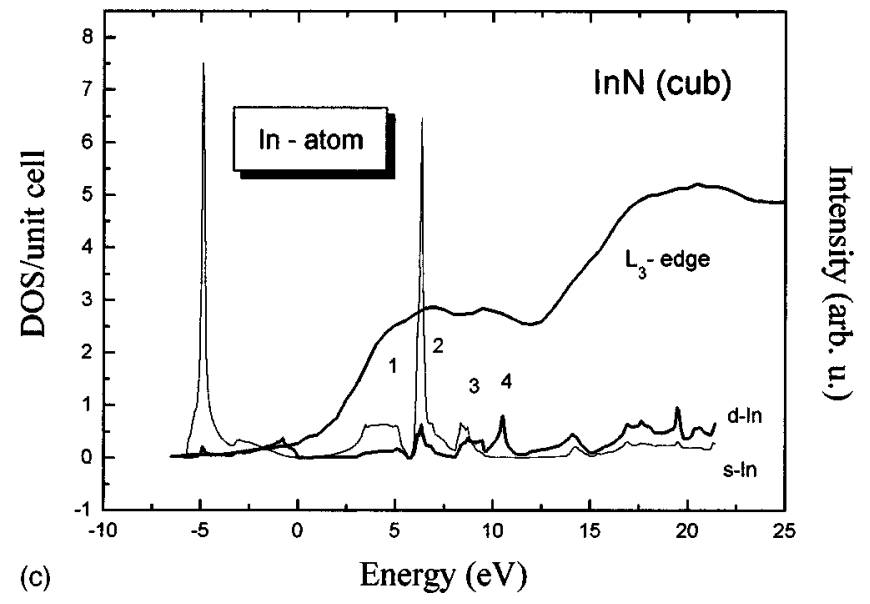

FIG. 6. The PDOS ( $s$, thin line; $p$, dotted line; $d$, thick line) as calculated for the cubic structure of InN. (a) Projected on the $\mathrm{N}$ atom and compared with the $\mathrm{N} K$ emission and absorption spectra. (b) Projected on the In atom: $p$ PDOS and $L_{1}$ absorption spectrum. (c) Projected on the In atom: $s$ and $d$ PDOS and $L_{3}$ absorption spectrum.

trast to the situation in $\mathrm{GaN}$, we have more $\sigma$ states close to the CB edge. Therefore, in ternary $\operatorname{In}_{x} \mathrm{Ga}_{1-x} \mathrm{~N}$ alloys with low In content, the states of InN $\sigma$ bonds can form states in the $c$ plane with energy located in the GaN energy gap.

For the spectra obtained at the $L_{1}$ edge of In [Fig. 5(b)] we did not resolve many details because the natural width of In $2 s$ level is $5 \mathrm{eV}^{26}$ However, overall agreement of the 
location of main maximum and minimum compares well with the calculated $p$ PDOS distribution. Comparing to $\mathrm{GaN}$, we find only the first and second peaks in PDOS are widely separated while the third and fourth peaks consist now of an assembly of a few other overlapping peaks.

In Fig. 5(c) measurements obtained at the $L_{3}$ edge of In together with the $s$ and $d$ PDOS of In are shown. The In $2 p_{3 / 2}$ level has a natural width of $2.65 \mathrm{eV}$ and limits the amount of details that can be resolved. The $\pi$ and $\sigma$ bonds are almost equally distributed; only at the third maximum in PDOS does the main contribution come from $\sigma$ bonds. Three well-separated peaks in $s$ PDOS are especially similar to those observed in GaN. The $d$ PDOS distribution is different. There is a considerably smaller contribution of $d$ states of cations to $\mathrm{CB}$ than in GaN or even AlN.

In the Fig. 6 the data for cubic InN are presented. At the $\mathrm{N}$ atom site [Fig. 6(a)] the PDOS and the emission and absorption spectra are very similar to those for GaN. Looking at the differences, they are only in the CB: the third maximum is weaker and the fourth maximum is now well resolved for both the $p$ and $d$ PDOS as well as for the experimental spectrum. At the cation site [Fig. 6(b)] the $p$ PDOS forms several weaker peaks and one sharp maximum nearly $10 \mathrm{eV}$, in very good agreement with the spectral shape obtained from the experiment. Although the $p$ PDOS peak distribution is different for the wurtzite and cubic cases [see Figs. 5(b) and 6(b)], the shape of experimental spectra is very similar (taking into account the $\sigma$ line in the wurtzite structure). The $s$ and $d$ PDOS distribution and also the measured spectrum at $L_{3}$ edge of In [Fig. 6(c)] are very similar to the wurtzite case and also somewhat similar to cubic GaN. The $s$ PDOS form a sharp peak at $6 \mathrm{eV}$, which is observed in the experiment; also the fourth peak from the $d$ PDOS is seen in the spectrum.

\section{BN}

The spectra for B in hexagonal $\mathrm{BN}$ have been already reported ${ }^{27-30}$ and discussed with respect to the core exciton structure seen in emission and absorption spectra. They were analyzed in terms of the contributions of $\pi$ and $\sigma$ bonds to the spectrum, but never directly compared with the DOS distribution. The "raw" DOS states projected on the N site along with the experimental $K$ emission and the $K$ edge of $\mathrm{N}$ (as measured for the polycrystalline hexagonal BN) powder are presented in Fig. 7(a). The natural width of the nitrogen $1 s$ orbital is below $0.1 \mathrm{eV}$ (Ref. 26) and the spectrometer resolution function is $\sim 0.16 \mathrm{eV}$ for absorption. Although the width of spectrum is close to the width of DOS and the position of main maxima in the spectrum agrees with the positions of main structures in DOS, the agreement is not so satisfactory as it was in the cases of GaN, AlN, and InN. In particular, the relative intensities of the characteristic features are somewhat different. One reason for differences between calculated and measured spectra is the preferred crystallite orientation with respect to the polarization vector of synchrotron radiation, which enhances the $\pi$-bonded states close to the VB and CB edges. ${ }^{29}$ Apart from the CB edge the $\sigma$ states dominate the spectrum. The three peaks located between 11 and $15 \mathrm{eV}$ are not resolved in the absorption spectrum. Another reason ${ }^{31}$ for the deviation between the obser-
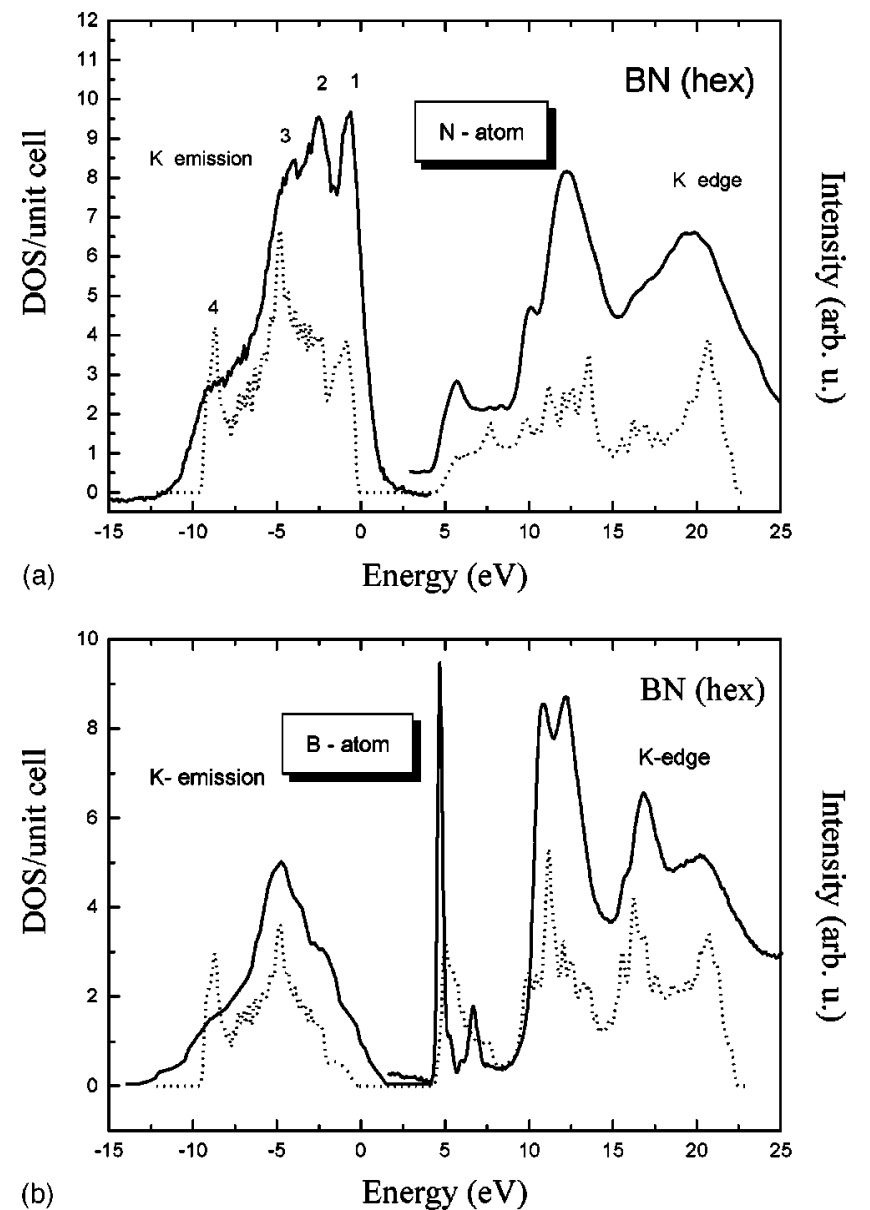

FIG. 7. The PDOS as calculated for the hexagonal structure of $\mathrm{BN}$ (dotted line) and compared with appropriate $K$ emission and absorption spectra (solid line). (a) Projected on the $\mathrm{N}$ atom. (b) Projected on the B atom.

vations and the calculations is incomplete screening of the core hole (the final state effect). The presence of a $1 s$ core hole in the initial state can shift the VB states closer to the VB edge and can be the reason for enhacement of the second maximum in the spectra. ${ }^{28}$ The anomaly in electron screening is not related to the nature of the $\mathrm{B}$ and $\mathrm{N}$ atoms but to the anisotropy of the bonds formed in the hexagonal structure, which can lead to incomplete screening of the core hole in this material. Moreover, it can be seen in powder samples due to preferential grain orientation.

The DOS distribution at the B site and the related spectra are presented in Fig. 7(b). In the emission spectrum the agreement of the calculated DOS distribution with that observed in the spectrum is very good, and it suggests that the core hole is screened efficiently in the case of the B atom. Differences between the calculated DOS and measured spectra are visible for states in the CB where the very pronounced pre-edge structure is observed and originates from transitions to $\pi$ orbitals. ${ }^{29}$ This structure is very characteristic for hexagonal $\mathrm{BN}$ and not observed for other nitrides.

Finally, in Fig. 8 the calculated PDOS and related measured spectra for cubic BN structure are shown. The agreement between calculations and experiment is quite good when we recall that the natural width of the $1 s$ B level is $0.07 \mathrm{eV}$ (Ref. 26) and the spectrometer function width is 0.2 


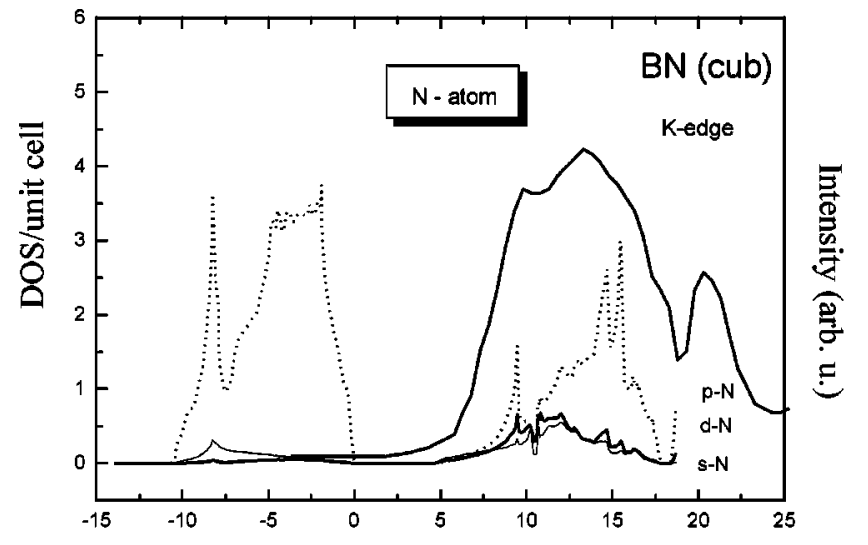

(a)

Energy (eV)

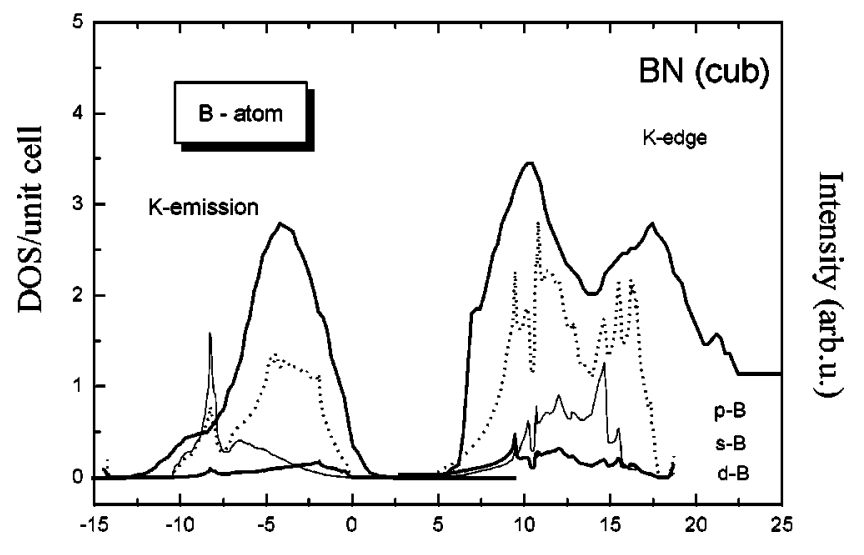

(b)

Energy (eV)

FIG. 8. The PDOS as calculated for the cubic structure of BN ( $s$, thin line; $p$, dotted line; $d$, thick line). (a) Projected on the $\mathrm{N}$ atom and compared with the N $K$ absorption spectrum. (b) Projected on the B atom and compared with B $K$ emission and absorption spectra.

$\mathrm{eV}$ for absorption and $0.5 \mathrm{eV}$ for emission spectra. The only deviation occurs at the $K$ edge of $\mathrm{B}$ close to the $\mathrm{CB}$ edge where the observed structure is absent in the calculated PDOS. In the cases of GaN, AlN, and InN there were close similarities between wurtzite and cubic structures. Now, we can see that hexagonal and cubic phases of $\mathrm{BN}$ are quite different regarding the calculated DOS and measured spectra. Moreover, these two phases of $\mathrm{BN}$ are not similar to wurtzite and cubic phases of previously considered nitrides. Also, one can notice much smaller hybridization between the states with different symmetry comparing to other nitrides.

\section{CONCLUSIONS}

The large set of the x-ray spectra for group-III nitrides with wurtzite (and BN hexagonal) and cubic structure are presented and discussed together with the "raw" PDOS energy distribution calculated using the LMTO method. In the case of GaN, AlN, and InN the agreement between the calculated partial density of states distribution and observed spectra is very good. This allows us to perform a detailed analysis of characteristic features of DOS, especially in context of partial DOS contributions to total DOS in VB and $\mathrm{CB}$. Moreover, some important conclusions about hybridization of $p$ - $d$ and $s-d$ states are drawn. General features of the spectra for $\mathrm{GaN}$ and $\mathrm{InN}$ are very similar, whereas some differences exist when we compare them with AlN. Thus can be explained by the lack of the cation semicore $d$ states in AlN.

Comparing the PDOS distribution between the wurtzite and cubic structure of considered compounds we also found close similarities. However, for the cubic structure more states are localized at the bottom of the VB, and in the $\mathrm{CB}$ the fourth peak is generally better separated and more visible than in the wurtzite structure.

Using the polarization properties of synchrotron radiation and well-defined orientation of our samples we were able to differentiate between states originating from $\sigma$ and $\pi$ bonds in the distribution of the PDOS. These measurements are new compared to the data reported by other authors for this class of materials. We detect pronounced differences between the distribution of the electron state in the $\mathrm{CB}$ for these two bonds as well at the $\mathrm{N}$ as at the cation site. The biggest anisotropy between $\sigma$ and $\pi$ bonds was found in AlN, which also has the largest difference in the corresponding lengths of bonds ( $b$ and $d$ in Table II). The observed anisotropy of the DOS distribution should appear in the anisotropy of various physical properties (refractive index, dielectric constant, elastic constants, etc.).

In contrast to the nitrides considered above, $\mathrm{BN}$ exhibits qualitatively different features. In particular, both calculated and measured spectra are quite different for cubic and hexagonal BN phases. Moreover, the agreement between calculated and experimental spectra for hexagonal $\mathrm{BN}$ is not as good as that for the previously considered nitrides. This can be explained by insufficient core hole screening, which is typical for insulating materials.

Considering the ionicity of investigated materials we analyze the ratio of anion to cation states in VB and $\mathrm{CB}$. The results are given in last two columns of Table I. More ionic bonding corresponds to higher valence electron density on the anion site, whereas the density of conduction states should be higher on the cation site. We found the ratio of anion to cation states in $\mathrm{VB}$ to be between $2 \mathrm{eV}(\mathrm{BN})$ and 4.6 $\mathrm{eV}(\mathrm{AlN})$. In the $\mathrm{CB}$ the differences are less pronounced, but indeed more states are located at the cation site for all considered nitrides. Except for AIN, the ionicity in the wurtzite (or BN hexagonal) structure was higher than that in the cubic structure. Generally, AlN and InN are more ionic than GaN, and $\mathrm{BN}$ has the lowest ionicity.

\section{ACKNOWLEDGMENTS}

The authors would like to thank P. Lagarde and R. Cortes for help in the measurements performed at LURE, Orsay, France, and S. Kul'kova for discussions. This work was partially supported by the State Committee for Scientific Research (Republic of Poland) Grants No. 2P03B 10114 and No. 3P03B 093 14. This work was also supported by National Science Foundation Grant No. DMR-9801804, the Science Aliance Center for Excellence Grant from the University of Tennessee, and a DOE-EPSCor cluster research Grant No. DOE-LEQSF (1993-95)-03. The Advanced Light Source is supported by the office of Basic Energy Sciences, U.S. Department of Energy under Contract No. DE-AC03-76Sf00098. 
*Electronic address: jablo@ifpan.edu.pl

${ }^{1}$ H. Sakai, T. Koide, H. Suzuki, M. Yamaguchi, S. Yamasaki, M. Koike, H. Amano, and I. Akasaki, Jpn. J. Appl. Phys., Part 2 34, L1429 (1995).

${ }^{2}$ S. Nakamura, M. Senoh, S. Nagahama, N. Iwasa, T. Yamada, T. Matsushita, H. Kiyoku, and W. Sugimoto, Jpn. J. Appl. Phys., Part 2 35, L74 (1996).

${ }^{3}$ V.A. Gubanov, Z.W. Lu, B.M. Klein, and C.Y. Fong, Phys. Rev. B 53, 4377 (1996).

${ }^{4}$ A. Loiseau, F. Willaime, N. Demoncy, G. Hug, and H. Pascard, Phys. Rev. Lett. 76, 4737 (1996), and references therein.

${ }^{5}$ M. Tanaka, S. Nakahata, K. Sogabe, H. Nakata, and M. Tabioka, Jpn. J. Appl. Phys., Part 2 36, L1062 (1997).

${ }^{6}$ T. Detchprohm, K. Hiramatsu, K. Itoh, and I. Akasaki, Jpn. J. Appl. Phys., Part 2 31, L1454 (1992).

${ }^{7}$ M. Leszczynski, H. Teisseyre, T. Suski, I. Grzegory, M. Bockowski, J. Jun, S. Porowski, K. Pakula, J.M. Baranowski, C.T. Foxon, and T.S. Cheng, Appl. Phys. Lett. 69, 73 (1996).

${ }^{8}$ N.E. Christensen and I. Gorczyca, Phys. Rev. B 50, 4397 (1994), and references therein.

${ }^{9}$ A. Rubio, J.L. Corkill, M.L. Cohen, E.L. Shirley, and S.G. Louie, Phys. Rev. B 48, 11810 (1993).

${ }^{10}$ W.R.L. Lambrecht and B. Segall, J. Rife, W.R. Hunter, and D.K. Wickenden, Phys. Rev. B 51, 13516 (1995).

${ }^{11}$ Yong-Nian Xu and W.Y. Ching, Phys. Rev. B 48, 4335 (1993).

${ }^{12}$ G. Martin, S. Strite, A. Botchkarev, A. Agarwal, A. Rockett, and H. Morkoc, Appl. Phys. Lett. 65, 610 (1994).

${ }^{13}$ C.B. Stagarescu, L.C. Duda, K.E. Smith, J.H. Guo, J. Nordgren, R. Singh, and T.D. Moustakas, Phys. Rev. B 54, R17 335 (1996)

${ }^{14}$ W.R.L. Lambrecht, S.N. Rashkeev, B. Segall, K. LawniczakJablonska, T. Suski, E.M. Gullikson, J.H. Underwood, R.C.C. Perera, J.C. Rife, I. Grzegory, S. Porowski, and D.K. Wickenden, Phys. Rev. B 55, 2612 (1997).

${ }^{15}$ K. Lawniczak-Jablonska, T. Suski, Z. LilientalWeber, E.M. Gullikson, J.H. Underwood, R.C.C. Perera, and T.J. Drummond, Appl. Phys. Lett. 70, 2711 (1997).
${ }^{16}$ L.C. Duda, C.B. Stagarescu, J. Downes, K. Smith, D. Korakakis, T.D. Moustakas, J. Guo, and J. Nordgren, Phys. Rev. B 58, 1928 (1998).

${ }^{17}$ Properties, Processing, and Applications of Gallium Nitride and Related Semiconductors, edited by J.H. Edgar, S. Strite, I. Akasaki, H. Amano, and C. Wetzel, EMIS Data reviews series No. 23 (1999).

${ }^{18}$ J.H. Underwood, E.M. Gullikson, M. Koike, P.C. Batson, P.E. Denham, and R. Steele, Rev. Sci. Instrum. 67, 1 (1996).

${ }^{19}$ J.J. Jia, T.A. Callcott, J. Yurkas, A.W. Ellis, F.J. Himpsel, M.G. Samant, J. Stohr, D.L. Ederer, J.A. Carlisle, E.A. Hudson, L.J. Terminello, D.K. Shuh, R.C.C. Perera, Rev. Sci. Instrum. 66, 1394 (1995).

${ }^{20}$ O.K. Andersen, Phys. Rev. B 12, 3060 (1975).

${ }^{21}$ N.E. Christensen, Phys. Rev. B 37, 4528 (1988).

${ }^{22}$ O. Jepsen and O.K. Andersen, Solid State Commun. 9, 1763 (1971).

${ }^{23}$ Numerical Data and Functional Relationships in Science and Technology, edited by O. Madelung, M. Schilz, and H. Weiss, Landolt-Bornstein, New Series, Group III, Vol. 17, Pt.a (Springer-Verlag, Berlin, 1982).

${ }^{24}$ H. Schulz and K.H. Tiemann, Solid State Commun. 23, 813 (1977).

${ }^{25}$ M. Methfessel, Phys. Rev. B 38, 1537 (1988).

${ }^{26}$ M.O. Krause and J.H. Oliver, J. Phys. Chem. Ref. Data 8, 329 (1979).

${ }^{27}$ E. Tegeler, N. Kosuch, G. Wiech, and A. Faessler, Phys. Status Solidi B 91, 223 (1979).

${ }^{28}$ A. Mansour and S.E. Schnatterly, Phys. Rev. B 36, 9234 (1987).

${ }^{29}$ J. Moscovici, G. Loupias, PH. Parent, and G. Tourillon, J. Phys. Chem. Solids 57, 1159 (1996).

${ }^{30}$ J.J. Jia, T.A. Callcott, E.L. Shirley, J.A. Carlisle, L.J. Terminello, A. Asfaw, D.L. Ederer, F.J. Himpsel, and R.C.C. Perera, Phys. Rev. Lett. 76, 4054 (1996).

${ }^{31}$ A. Chaiken, L.J. Terminello, J. Wong, G.L. Doll, and C.A. Taylor, Appl. Phys. Lett. 63, 112 (1993). 